\title{
The importance and challenge of pediatric trials of hemophilia drugs
}

\section{To the Editor:}

In the February Opinion "Pediatric requirements in Europe stymie help for hemophilia," Peyvandi et al. ${ }^{1}$ challenged the European requirements for the development of recombinant and human plasma-derived factor VIII (FVIII) and factor IX (FIX) products in children. In their view, the sequential clinical trial process for FVIII and FIX products as defined in the scientific guidelines ${ }^{2,3}$ of the European Medicines Agency (EMA) will delay the availability of new products for adults in the European Union. Similar considerations by one of the authors ${ }^{4}$ have already been addressed elsewhere ${ }^{5}$.

Hemophilia is a disease that in the past typically affected only children, as the vast majority of affected individuals never reached adult age; it became a greater concern in adults only after the development of effective and safe treatments ${ }^{6}$. Considering this, the EU guideline requests that sufficient pediatric data be available when a new medicinal product for hemophilia is submitted for marketing authorization. This approach ensures that in cases of a significant pediatric medical need, the target pediatric population is not deprived of high-quality, safe and effective medicinal products.

The authors pointed out an urgent need for long-acting coagulation factors, which would reduce the frequency of intravenous administrations and may be less immunogenic than other treatments. Although less frequent administrations certainly would benefit patients, many safe and effective FVIII and FIX products are already authorized in the European Union for adult and pediatric patients with hemophilia. Newer products with a longer half-life are potentially more interesting for prophylactic use rather than on-demand use and are consequently more relevant for pediatric use because prophylactic administration is more common in children.

The EU requirement to include in the pediatric studies a minimum number of doses does increase the duration of studies for long-acting products, which are administered less often. However, this requirement is needed to evaluate the potential for immunogenicity, which is a major risk factor in the treatment of hemophilia $\mathrm{A}$, more so in children than adults.

Consequently, timely access in adults has to be balanced with the need to assess the safety of a product before it is used in children. The EMA has demonstrated flexibility in its approach to balance these competing

\section{To the Editor:}

A recent Opinion piece by Peyvandi et al. ${ }^{1}$ was critical of the European requirement that pediatric data on hemophilia medicines be included in initial marketing authorization applications (MAAs). The passion the authors expressed for expediting the availability of new treatments for adult patients is commendable. However, the Opinion may leave the false impression that the source of the requirement for pediatric data before drug approval is the European Paediatric Regulation and the associated mandate for a Paediatric Investigation Plan (PIP). In actuality, the Paediatric Regulation is not the basis for requiring that pediatric data for hemophilia products requirements in the guidelines, for example, by requesting inclusion of only a limited number of pediatric patients in the studies and particularly by specifying that more than half of the required doses in pediatric studies can be administered after marketing authorization has been requested in adults. Such an approach aims to prevent unnecessary delays in authorization.

Commencing the requested pediatric trials as early as possible in the overall medicinal product development process is therefore the key to avoiding or reducing the delay of marketing authorization.

The use of new medicinal products in children should rely on sufficient evidence for pharmacokinetics, safety and efficacy in all age groups. Experience has shown that pharmaceutical companies encounter considerable difficulties when trying to enroll children and complete pediatric studies once a medicinal product is available on the market for adults because of widespread off-label use in children.

We strongly believe that with appropriate planning, including early interaction with regulators, it is possible to reconcile timely product access for adults with sufficient information to support the treatment's use in children.

\section{COMPETING FINANCIAL INTERESTS}

The authors declare competing financial interests: details are available in the online version of the paper (doi:10.1038/nm.3546).

Dirk Mentzer ${ }^{1,2}$, Emilie Desfontaine ${ }^{1} \&$ Paolo A Tomasi ${ }^{1}$
${ }^{1}$ European Medicines Agency, London, UK. ${ }^{2}$ Paul-Ehrlich Institute, Langen,
Germany.
e-mail: paolo.tomasi@ema.europa.eu

1. Peyvandi, F., Rosendaal, F.R., O'Mahony, B. \& Mannucci, P.M. Nat. Med. 20, 117 (2014).

2. European Medicines Agency. European Medicines Agency, http://www.ema.europa. eu/docs/en_GB/document_library/Scientific_guideline/2011/08/WC500109692.pdf (2011).

3. European Medicines Agency. European Medicines Agency, http://www.ema.europa. eu/docs/en_GB/document_library/Scientific_guideline/2011/08/WC500109691.pdf (2011).

4. Mannucci, P.M. Haemophilia 19, 344-348 (2013).

5. Hilger, A. et al. Haemophilia 19, 349-350 (2013).

6. Jones, P.K. \& Ratnoff, O.D. Ann. Intern. Med. 114, 641-648 (1991).

be included in the initial MAA.

The Paediatric Regulation requires that medicines developed to treat indications that include a pediatric population must be studied in children, not just adults, and that the pediatric studies must be conducted in accordance with an approved PIP. The regulation also authorizes companies to defer the start of pediatric studies so that approval of the medicine for adults is not delayed, a point that Peyvandi et al. acknowledge yet appear to believe is not being utilized. The authors criticize the Paediatric Regulation and PIP requirement along with the European Medicines Agency (EMA) Blood Products Working Party (BPWP) guidelines on clinical investigations of factor VIII and factor 